\title{
Global mapping of live cell mechanical features using PeakForce QNM AFM
}

\author{
Jinrong $\mathrm{Hu}^{1,2}$, Shenbao Chen ${ }^{1,2}$, Dandan Huang ${ }^{1,2}$, Yan Zhang ${ }^{1,2}$, Shouqin Lü $^{1,2 \bowtie}$, Mian Long ${ }^{1,2 \bowtie}$ \\ ${ }^{1}$ Center of Biomechanics and Bioengineering, Key Laboratory of Microgravity (National Microgravity Laboratory), \\ Beijing Key Laboratory of Engineered Construction and Mechanobiology, and CAS Center for Excellence in Complex \\ System Mechanics, Institute of Mechanics, Chinese Academy of Sciences, Beijing 100190, China \\ 2 School of Engineering Science, University of Chinese Academy of Sciences, Beijing 100049, China
}

Received: 12 June 2019 / Accepted: 25 September 2019 / Published online: 22 January 2020

\begin{abstract}
Atomic force microscopy (AFM) has been widely used to acquire surface topography upon different scanning modes and to quantify mechanical properties of a cell using single-point ramp force mode. However, these traditional measurements need massive force curves originating from multiple points of a cell to exclude the potential errors resulted from limited and factitious selections of testing points, making the measurements time-consuming and highly localized. PeakForce Quantitative NanoMechanics (PF QNM) is a high-speed (faster by 3-4 order of magnitude) and global surface mechanical property mapping method with high spatial resolution, overcoming the drawbacks of traditional ramp mode especially used for a live cell with high heterogeneity. In this protocol, we elaborated how to run PF QNM measurements for live cells and relevant modification may be needed when extending this method to other cell-like soft materials.
\end{abstract}

Keywords Global mechanical mapping, Live cell, Atomic force microscopy, PeakForce QNM

\section{INTRODUCTION}

Mechanical features of cells are significant to cellular behaviors and fate decision in many biological processes such as cell migration, growth, and differentiation responses to mechanical stimuli (Calzado-Martín et al. 2016; Geitmann and Ortega 2009; Qi et al. 2017; Wang et al. 2014; Xu et al. 2019). Thus, global and high-speed mechanical property mapping with high spatial resolution is important for understanding the dynamic behaviors of cells. Many approaches like magnetic tweezer, micropipette aspiration, cone plate viscometer and atomic force microscopy (AFM) are optional for cell mechanical measurements with respective advantages (Long et al. 2011; Rodriguez et al. 2013). AFM has been used widely with its mature operation and analysis

$\bowtie$ Correspondence: lsq@imech.ac.cn (S. Lü), mlong@imech.ac.cn (M. Long) system in the following two aspects of applications. Firstly, it has been used to acquire material surface topography, chemical and electrical properties upon different scanning modes (Barbee et al. 1994; Maivald et al. 1991; Zhong et al. 1993) and special probes (Rawlett et al. 2002; Sugimoto et al. 2007). Secondly, single-molecule force spectrum AFM upon ramp mode empowers us to investigate protein folding/unfolding and ligand-receptor interactions (Le et al. 2017; Marshall et al. 2003). In addition, localized mechanical properties of a cell or cells can also be extracted from corresponding force-distance curves of special sites based on contact mechanics theories (Sneddon 1965). Nowadays, AFM achieves its highest resolutions to subnanometer of scanning and picoNewton ( $\mathrm{pN}$ ) of force, makes it possible to resolve even atomic arrangement and interactions (Sugimoto et al. 2007).

With the development of AFM technology, more and more detecting modes are exploited. Taking mechanical 
property acquisition as an example, Force volume (FV) mode AFM performs mechanical mapping of a cell or cells through combining topography scanning with single-point force-distance curve arrays in the selected ranges (Hansma et al. 1997; Radmacher et al. 1994). FV mode is a basic mode for all AFM instruments with its robustness in force-distance curve acquisition but is time-consuming with low sampling speed (usually $<10 \mathrm{~Hz}$ ). Fast force volume (FFV) and quantitative imaging (QI) modes are thus developed based on FV mode for much faster force-distance curve acquiring. While FFV or QI mode can map global mechanical property of a cell or cells with the advance of sampling speed and the spatial resolution of global mechanical property, accurate force control is still limited in those modes. PF QNM (PeakForce Quantitative NanoMechanics) is another mode released by Bruker for fast mechanical mapping based on PF tapping mode. With better force control than FFV and QI modes, PF QNM mode enables more precise global mechanical mapping with high spatial resolution and speed (see details in the following section "PRINCIPLES").

Nowadays, PF QNM mode has been attracting attentions in biological issues upon its unique advantages. For example, it is well applied in characterizing dynamic changes of the elastic modulus of plant leaves (Qi et al. 2017) as well as in extensive applications in plant biomechanics. In recent years, PF QNM mode has also been extended to map global surface topography and mechanical features of mammalian live cells (Pittenger and Slade 2013; Schillers et al. 2016), including isolating tumorigenic cells from normal cells (Calzado-Martín et al. 2016) and investigating mechanosensing of neutrophils onto HUVEC monolayer pre-cultured on stiffness-varied substrates (Xu et al. 2019). Here we present all the detailed protocols from experimental performance to data analysis in running PF QNM measurements for live cells. Actually, this protocol can also be used to perform mechanical measurements for other soft materials with suitable settings.

\section{MATERIAL, EQUIPMENT AND SOFTWARE}

\section{Cell preparation}

Human umbilical vascular endothelial cell line (HUVEC) purchased from ATCC (American Type Culture Collection. Manassas, VA, USA) was used to represent typical mechanical mapping of live cells. HUVECs were cultured in Medium 199 supplemented with 20\% FBS, $100 \mathrm{U} / \mathrm{mL}$ penicillin, $20 \mathrm{mmol} / \mathrm{L}$ HEPES (HyClone, Logan, Utah, USA), $3 \mu \mathrm{g} / \mathrm{mL}$ thymidine (Sigma-Aldrich, Shanghai,
China), $1 \mathrm{mmol} / \mathrm{L}$-glutamine (Sigma-Aldrich, Shanghai, China), $14 \mathrm{U} / \mathrm{mL}$ heparin sodium (Sigma-Aldrich, Shanghai, China), $25 \mu \mathrm{g} / \mathrm{mL}$ amphotericin B (Amresco, Solon, OH, USA), and $5 \mathrm{ng} / \mathrm{mL}$ bFGF (R\&D Systems, Minneapolis, MN, USA). They were plated on collagen I (Sigma-Aldrich, Shanghai, China) coated glass bottom dishes to form a monolayer before using.

\section{Equipment}

- AFM: Bioscope Catalyst (Bruker, Billerica, MA, USA) with PF QNM module installed.

- AFM probes: Pre-calibrated PFQNM-LC probes (Bruker, Billerica, MA, USA) with spring constant of $\sim 0.07 \mathrm{~N} / \mathrm{m}$ were used for mechanical mapping. Tip parameters are in a height of $17 \mu \mathrm{m}$ and a tip radius of $65 \mathrm{~nm}$. For comparison, a spherical probe (NOVASCAN, Boone, IA, USA) with a nominal spring constant of $0.07 \mathrm{~N} / \mathrm{m}$ and a tip radius of $2.5 \mu \mathrm{m}$ and a pyramid MLCT-E probe (Bruker, Billerica, MA, USA) with a nominal spring constant of $0.03 \mathrm{~N} / \mathrm{m}$, a tip height of $2-8 \mu \mathrm{m}$ and a tip radius of $20 \mathrm{~nm}$ were used for live cells in a conventional ramp mode.

\section{Software}

- NanoScope 8.15 (Bruker, Billerica, MA, USA): online software for running the measurements.

- NanoScope analysis v1.80 (Bruker, Billerica, MA, USA): offline software for performing data analysis.

- MATLAB (MathWorks, Natick, MA, USA): software for user-defined data analysis codes (available at https:// github.com/chenshenbao/AFM_PeakForce_QNM_mapping data_analysis).

- Prism 6 (GraphPad, San Diego, CA, USA): software for statistical analysis and graph drawing.

\section{PRINCIPLES}

\section{Principle of AFM test}

Schematic of working principle of an AFM device is shown in Fig. 1A. A piezoelectric translator (PZT) is used to drive a probe, composed by an elastic cantilever and a tip, approaching to, contacting with, and retracting from the sample. The interaction between the sample and the tip is characterized by the product of the deformation and spring constant of cantilever, and the deformation of cantilever is quantified by the deflection of the reflected laser from its back through a quad photodetector (QPD). For surface topography scanning, 


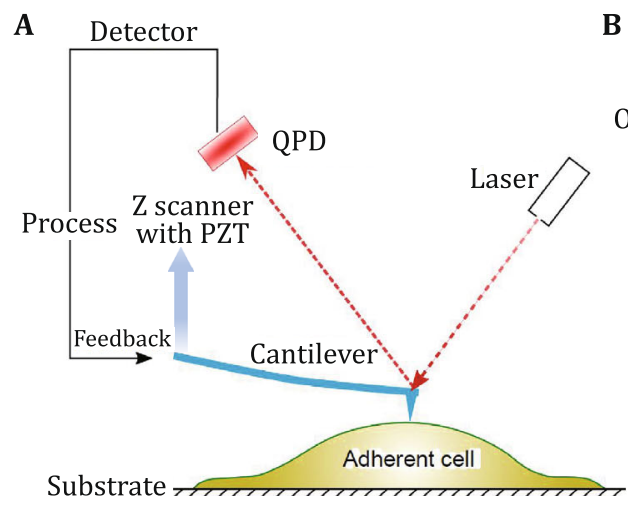

E

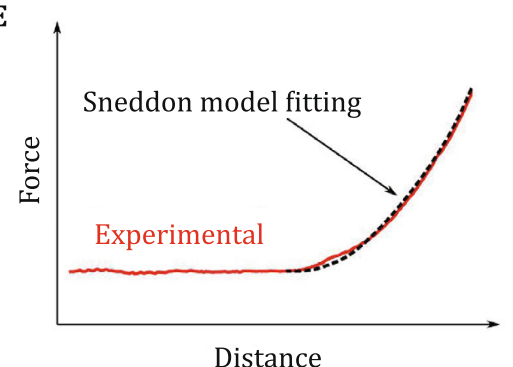

B

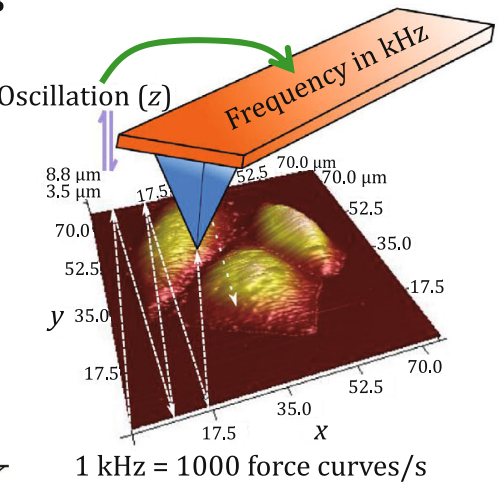

C

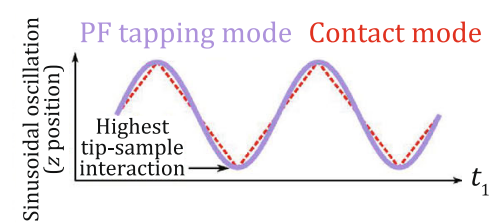

D

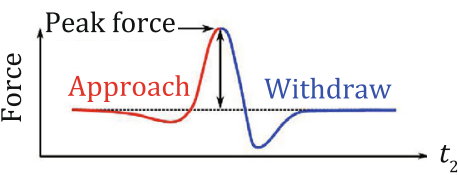

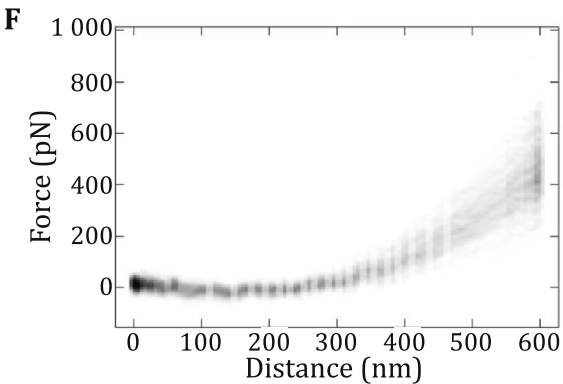

G

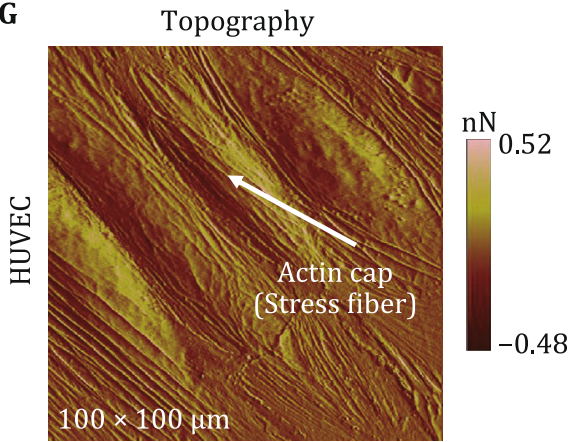

H

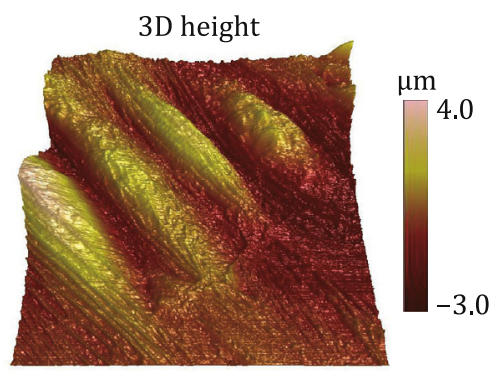

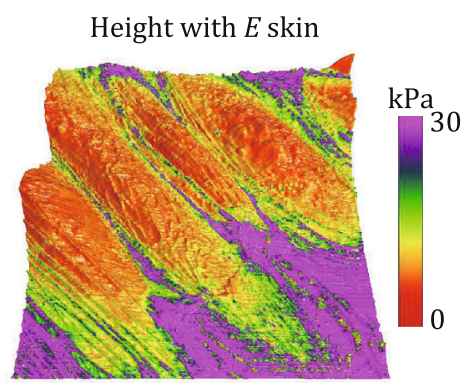

Fig. 1 Schematics of AFM method and PF QNM scanning mode. A Schematic of an AFM test. B Schematic of PF QNM scanning mode. C Difference of tip trajectories between PF QNM and ramp modes. D Typical force-time curve of a single approach-contact-withdrawal circle. E Force-distance curve fitting upon Sneddon model with red line for experimental data and black line for the fitted prediction. F Density plot of thousands of force curve obtained in one PF QNM scanning run. G-I Representative PF QNM mapping images of HUVECs for topography (G), 3D height $(\mathbf{H})$ and height with $E$ skin (I), respectively

different scanning modes determine distinct criterions for controlling PZT movement upon feedback loop. For example, the criterion of a contact scanning mode is to apply a constant force between the tip and the sample. Thus, the deflection of the cantilever on each scanning site is first adjusted to be equal to the pre-set setpoint through the $Z$-axis movement of PZT and then move to the next scanning site, where the $Z$-axis displacement of each site is recorded as the representation of surface height. For force-distance curve acquisition, the tip is driven to approach to, contact with, and retract from the sample surface along $Z$-axis upon the pre-setting of contact force, and both the approach and retraction curves are recorded for further analyses. AFM device adopts a classical PID (Proportional-Integral-Derivative) feedback system to adjust the movement errors and track the sample surface accurately (Åström and Hägglund 1995).

\section{Principle of PF QNM mode}

PF QNM is based on Bruker patented technology of PF tapping released in 2009 and the most dominant feature of this mode is high-speed surface mechanical mapping. In this mode, $Z$-axis tip engagement is driven by the sinusoidal oscillation of the PZT with high frequency of $0.25-8 \mathrm{kHz}$ (Fig. 1B), resulting in faster force-distance curve acquisition by 3-4 order of magnitude than that 
of classical ramp mode. The sinusoidal oscillation of the PZT in PF tapping mode also differs in its tip engaging trajectory along $Z$-axis from that of ramp mode. As shown in Fig. 1C, the PZT in ramp mode drives the tip moving up and down in fixed velocity (red line), resulting in high approaching velocity of the tip to the surface. Alternatively, the PF tapping mode enables the tip contact the sample surface in the valley of sinusoidal oscillation with approaching velocity of zero (purple line), protecting the sample with less damage. Furthermore, the oscillation frequency of the cantilever in PF tapping mode is far lower than the resonance frequency, keeping the stable tip engagement and the precise control of the indentation force (Fig. 1D). Above features of PF QNM enable this technology to be used for global mechanical mapping of a cell or cells with similar spatial resolution with that of surface topology scanning.

\section{Young's modulus fitting}

Different theoretical models are developed for extracting mechanical property of the sample from AFM forcedistance curve. Sneddon model (Sneddon 1965) is used mostly to fit the curve for getting Young's modulus $E$ upon pyramid tip (Fig. 1E):

$F=\frac{2}{\pi} \frac{E}{1-v^{2}} \tan \theta \delta^{2}$,

wherein $F$ denotes interaction force between tip and sample, $v$ is Poisson's ratio, $\theta$ defines half angle of the tip and $\delta$ is indentation depth. Hertz model is used for fitting when a spherical probe is chosen:

$F=\frac{4}{3} \frac{E}{1-v^{2}} \sqrt{R} \delta^{3 / 2}$,

wherein $R$ denotes the tip radius. Additionally, Hertzbased Derjaguin-Muller-Toropov (DMT) model is preferred when adhesion forces between the tip and the specimen are taken into consideration (Derjaguin et al. 1975):

$F=\frac{4}{3} \frac{E a^{3}}{R}+F_{\mathrm{adh}}$,

wherein $\alpha$ denotes the contact radius and $F_{\text {adh }}$ is the adhesion force $\left(F_{\mathrm{adh}}=-2 \pi R \Delta \gamma\right.$ and $\gamma$ is the surface energy). As spherical probes are congenitally unsuitable to resolve the detailed surface topography and perform global mechanical mapping with poor spatial resolution, pyramid probes are used to run PF QNM measurements and Sneddon model is adopted to implement force-distance curve fitting in this protocol. Using PF QNM mode can collect several tens of thousands of force-distance curves for each run, as the density plot shown in Fig. 1F, and Young's modulus for each point is extracted by fitting corresponding forcedistance curves. It should be noted that the exact number of force-distance curves collected depends on the scanning parameter settings of scan rate, sample/line and PeakForce frequency.

Representative surface topography, 3D height image and height with $E$ skin image of HUVECs were shown in Fig. 1G-I, respectively. Actin stress fibers at cell peripheries were easy to be discerned, and actin cap, namely stress fibers over the top of the nucleus, could also be found from the surface topography (Curry et al. 2017; Efremov et al. 2018), even though fluorescence labeling is usually required to confirm the existence of actin bundles. The mechanical distributions of the whole scanning range were also visualized in $E$ skin image with larger $E$ values in the cell periphery and the cell-cell junctions than those of cell bodies. Thus, PF QNM mapping makes it possible to quantify the differences in mechanical properties of distinct cell regions with the same spatial resolution of scanning, which may be helpful for exploring the elaborate mechanical features at subcellular level especially for the highly heterogeneous cell.

\section{PROTOCOL}

The summary of the entire procedure is listed in Fig. 2.

\section{Cell preparation}

Firm adhesion of cells to substrate is necessary for performing PF QNM mapping. Generally, cells are seeded on the substrate and cultured at least overnight onto the dishes to ensure the firm adhesion. In addition to special culture medium for specific cell types, poly-L-lysine (PLL)-coated glass bottom dishes are also preferred for cell adhesion. The cells are washed 2-3 times using pre-warmed DPBS (or any other buffer suitable or even culture mediums) before AFM imaging for reducing tip contamination and laser interference.

\section{Equipment pre-settings}

(1) Turn on the computer and controller $\rightarrow$ (2) Mount the probe on the holder and mount the holder onto the detector $\rightarrow$ (3) Open the online software NanoScope 8.15 (or other versions) and choose PF QNM mode $\rightarrow$ (4) Initialize the stage $\rightarrow$ (5) Align the laser to the suitable site of back of the cantilever for a maximum 


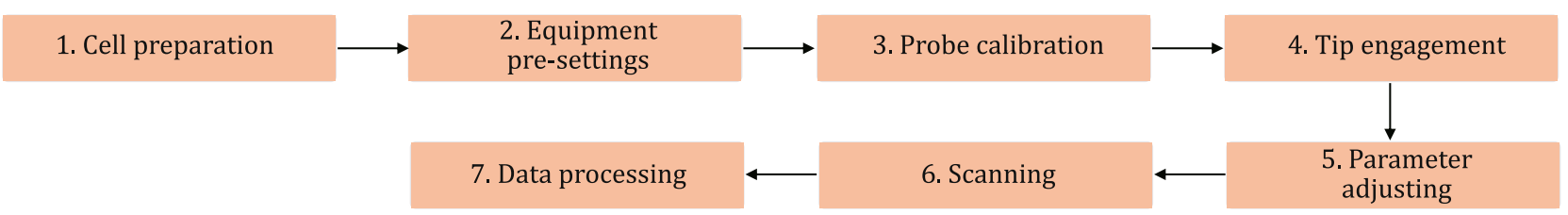

Fig. 2 Flowchart for PF QNM mapping

laser SUM value $\rightarrow$ (6) Adjust the laser point. Technically, it is favorable to adjust PeakForce frequency to $0.5 \mathrm{kHz}$ (or even lower to $0.25 \mathrm{kHz}$ ) for getting stable force-distance curves and set PeakForce amplitude to $300 \mathrm{~nm}$ for withdrawing the probe sufficiently from the sticky cell surface in each test circle.

\section{Probe calibration}

Different probes possess distinct inherent properties such as spring constant $k$ and deflection sensitivity (DS). To ensure the consistency of measurements, the probe must be calibrated in situ before test even though the nominal spring constant is given. There are two kinds of relative and absolute methods for probe calibrating. The relative calibration depends on standard sample with known Young's modulus, and the mechanical property of the sample to be tested should be comparable with that of standard sample. In general, the relative calibration method is suitable for measuring hard materials. Since the live cell is soft, the alternative absolute method is suitable for calibrating the probes with following steps: (1) Mount the cell dish onto the stage and then assemble the detector to the stage. (2) After aligning the laser point, calibrate DS first by indenting the probe to an extremely hard sample surface (like glass or sapphire) and then go to thermal tune panel (calibrate $\rightarrow$ thermal tune bottom for Bruker device) for $k$ calibration. It should be noted that the laser point may not be stable with leaping around at beginning because of the thermal gradient between the probe and the culture medium, resulting that the calibration needs to be performed after the laser drifting through 5-10 min waiting. And the operator may also lower the probe closer to the sample surface before calibration using navigation in the control panel to save time in the followed engagement. To image the live cells stably, it is highly recommended to choose PFQNM-LC probe with pre-calibrated spring constant $k$.

When using PFQNM-LC probes, DS can be backcalibrated using thermal tune based on energy equipartition theorem. As the probe can be treated as a harmonic oscillator, the mean kinetic energy of each degree of freedom (DOF) of the cantilever equals $k_{\mathrm{B}} T / 2$, wherein $k_{\mathrm{B}}$ is the Boltzmann constant and $T$ is the thermodynamic temperature. Since the potential energy of the entire cantilever is proportional to the kinetic energy of each DOF, we can derive approximately,

$\frac{1}{2} k_{\mathrm{B}} T \sim \frac{1}{2} k x^{2}$,

wherein $x$ denotes the deformation of the cantilever. Supposing that one applies two runs of thermal tune using the same probe and the first run has false $\mathrm{DS}_{1}$ then gives wrong $k_{1}$ but the second run has real $\mathrm{DS}_{2}$ and gives real $k_{2}$, the energy in each run is still the same and yields,

$\frac{1}{2} k_{\mathrm{B}} T \sim \frac{1}{2} k_{1} x_{1}^{2}=\frac{1}{2} k_{2} x_{2}^{2}$,

in which $x=\mathrm{DS}$. DF with DS in $\mathrm{nm} / \mathrm{V}$ and DF (deflection) in $\mathrm{V}$. Then we have:

$\left\{\begin{array}{l}x_{1}=\mathrm{DS}_{1} \cdot \mathrm{DF}_{1} \\ x_{2}=\mathrm{DS}_{2} \cdot \mathrm{DF}_{2}\end{array}\right.$.

Substitute Eq. 6 into Eq. 5 and consider the same probe with same driving force, the deflection of the cantilever should be the same as $\mathrm{DF}_{1}=\mathrm{DF}_{2}$ and then reads:

$k_{1} \mathrm{DS}_{1}^{2}=k_{2} \mathrm{DS}_{2}^{2} \rightarrow \mathrm{DS}_{2}=\mathrm{DS}_{1} \sqrt{\frac{k_{1}}{k_{2}}}$.

Prior to the measurements, the operator can set real value of $k$ as $k_{2}$ in thermal tune panel, and then assign an arbitrary/false DS as DS ${ }_{1}$. After running the thermal tune, one can get a fitted false $k$ as $k_{1}$ and the real DS (namely $\mathrm{DS}_{2}$ ) of the cantilever can be obtained using Eq. 7. In addition, one can also use RS-15 M sample by Bruker (or any other roughness sample) to calibrate the tip radius before measurement. Constant-temperature incubator mounted on the stage may be used for those cells sensitive to temperature changes.

\section{Tip engagement}

Set the scan size to a small square region (mostly with the length of side $\leq 30 \mu \mathrm{m}$ ), scan rate to a small value (mostly $<0.5 \mathrm{~Hz}$ ), and sample/line to $128 \times 128$ or $256 \times 256$ pixels at first, collectively ensuring that the lateral tip velocity is set mostly $\leq 30 \mu \mathrm{m} / \mathrm{s}$ and the 
tip/sample is in less damage and less likely to crash. Then click engage on the control panel and the probe is forced to approach the cell surface. The operator can enhance the parameter of engage setpoint to help the probe find the surface easily. Here the default value of engage setpoint is $0.3 \mathrm{~V}$ and $0.1 \mathrm{~V}$ is always a good choice in practice. This is because the extremely higher engage setpoint value could damage the sample due to the larger contact force applied. When the tip is not able to find the surface, a false engage error is reported to indicate the quite low engage setpoint value.

\section{Parameter adjusting}

Once the tip finds the surface, scanning starts right after a short duration of device self-adjusting (about $20 \mathrm{~s}$ ). Set the parameters of ScanAsyst auto control as individual and of ScanAsyst auto setpoint (SAS) as Off. Then adjust manually the tip-sample interaction force (in $\mathrm{pN}$ or $\mathrm{nN}$ ), namely, SAS. Increase SAS value gradually to obtain well-defined force-distance curves (Fig. 1E), usually ranging from $200 \mathrm{pN}$ to $1 \mathrm{nN}$ for live cell mechanical imaging. Note that SAS value depends on the measurement site and depth, one may increase/ decrease SAS value to capture the mechanical behaviors of deeper/shallower layer of the cell. Then adjust gradually scan size to the assigned size (mostly $<100 \mu \mathrm{m}$ on Catalyst). Note that the golden standard to judge if a SAS value is suitable is to monitor the quality of the force curves showing on the force monitor. Here this value is cell-type specific with a low value for flat fibroblasts but a high value for rugged HeLa cells and probe specific from one to another. In some cases, it is optional to manually tweak feedback gain for minimizing the peak force error and ensuring the tip track the surface correctly. Again, always keep the lateral tip velocity $\leq 30 \mu \mathrm{m} / \mathrm{s}$ to avoid tip crash error. Decrease scan rate to a lower value when a tip crash error reports.

\section{Scanning}

After completing the parameter settings, click frame down/up to initialize a target scanning. Make sure that the height, peak force error, Sneddon modulus and deformation channels are chosen. Notes: (1) Do not change any scanning parameters once the scanning starts to prevent the data recorded incorrectly; (2) When some errors like tip crash are encountered (without tip withdrawal), the operator can use capture now to capture the data manually.

\section{Data processing}

The data obtained from the online software are then analyzed using either the official offline software of NanoScope analysis or in-house programmed MTALAB codes (Fig. 3).

Processing using NanoScope analysis (Fig. 3A-C). After opening the data file to be processed in the software, one can click the Sneddon modulus (Young's modulus $E$ fitting using Sneddon model) channel as shown in Fig. 3A. To clarify $E$ distribution along an arbitrary cross-section, one can use section tool on the toolbar to draw the arbitrary horizontal lines (white line in Fig. 3A) for producing various cross-sections, and the typical $E$ values along the section are shown in Fig. 3B. This is a convenient way for users to exhibit quantitatively $E$ distributions of a cell with different structures or regions. For example, the $E$ values along cell-cell junctions (blue arrow) are much higher than those of stress fibers (cyan arrow) (Fig. 1I), consistent with the feature that cell-cell junctions are stiffer than the cell body or stress fibers across the nucleus (Ohashi et al. 2002; Vargas-Pinto et al. 2013). It is noticed that the nominal stiffness of the nucleus measured in this protocol is the stiffness of the cytoplasm/cortex right above the nucleus with the indentation depth of about $400 \mathrm{~nm}$, but not that of the nucleus itself. The actual stiffness of the nucleus should be measured upon deeper indentation even penetrating cell membrane and touching nucleus membrane directly (Liu et al. 2014). Also, one can use particle analysis tool to do the statistics of $E$ in freely-drawn boxed regions (Fig. 3A, white dashed box). The software runs the statistics of each data points (one point one $E$ value) inside the box and presents the results in frequency distributions in Fig. 3C, which can be used to calculate the mean $E$ value for the region-of-interest (ROI) (Qi et al. 2017). In this case, one should be careful to select the parameter $X$ axis in statistical histogram of $E$ inside the box, where absolute but not relative must be chosen for correct data export (for Bruker offline processing software NanoScope analysis only).

Processing using MATLAB (Fig. 3D-F). While the official offline software works well for conventional analyses and data extraction, only regular rectangular or square boxes can be drawn using the software and this limitation confines the elaborative analyses of any other ROIs with irregular boundary. Thus, we programmed MATLAB codes to pick out each $E$ value in amorphous or closed regions (Fig. 3D) and to circle out the ROIs for extracting corresponding $E$ values for further analyses (Xu et al. 2019). As an example, the two circled white contours in Fig. 3D defined cell body and junction 
A
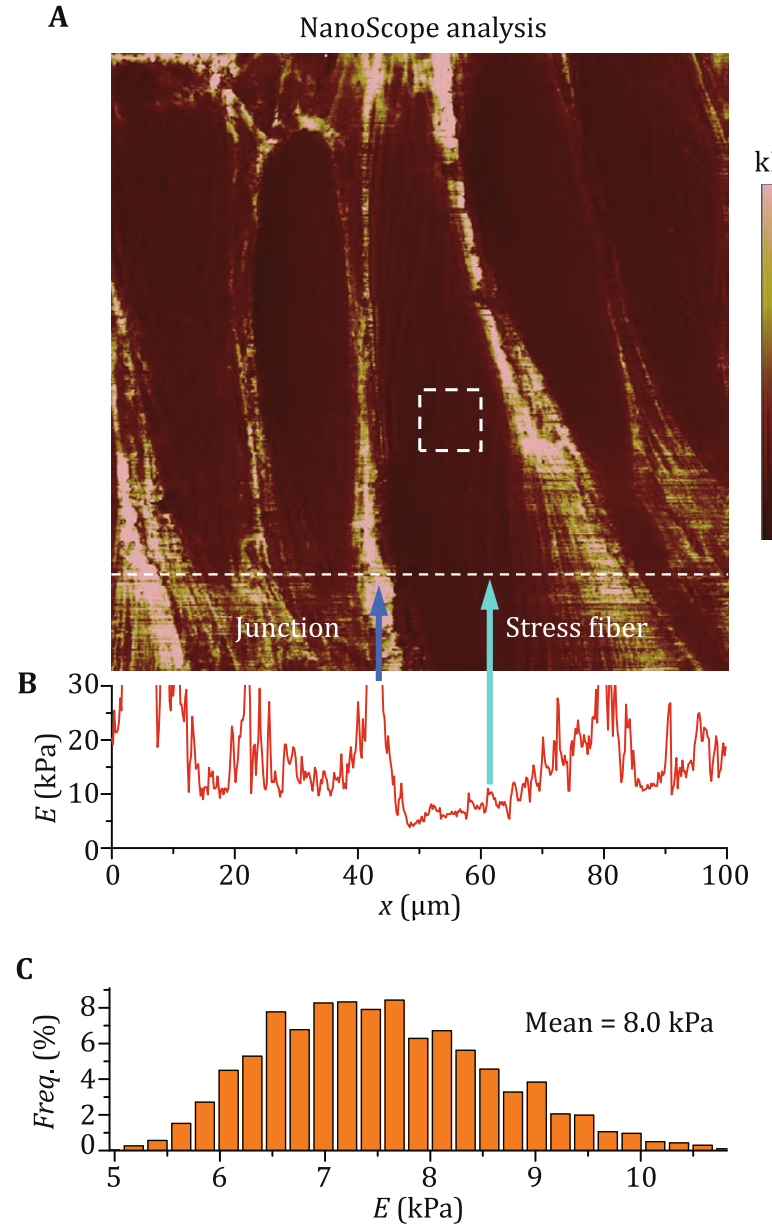

D

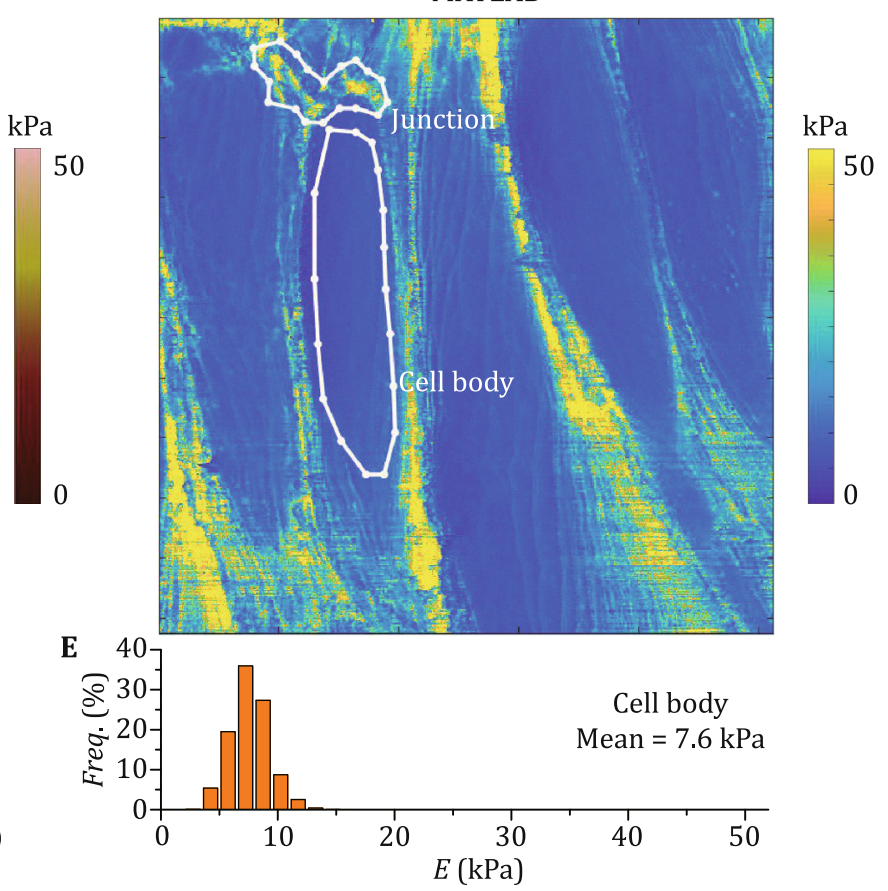

$\mathbf{F}$

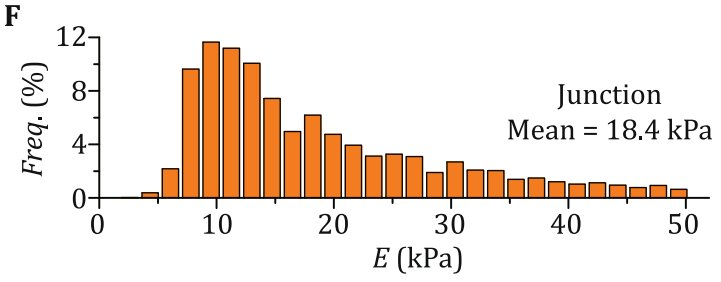

Fig. 3 Data analysis for PF QNM images. Data are extracted and analyzed using offline software NanoScope analysis (A-C) or in-house programmed MATLAB codes (D-F). A, D Representative Young's modulus $E$ mapping results for HUVECs upon NanoScope analysis (A) and MATLAB codes (D), respectively. B $E$ values along the white dashed line of A. C Frequency (Freq for short) distribution of $E$ values inside the white dashed box of A. E, F Frequency distributions of $E$ values inside irregularly-circled cell body (E) and junction (F) regions of $\mathbf{D}$

regions and the corresponding statistics of $E$ distributions of these two regions were shown in Fig. $3 \mathrm{E}$ and $\mathrm{F}$, respectively. Apparently, the junction region is much stiffer with right shifted $E$ distribution and higher mean value of $18.4 \mathrm{kPa}$ than those of the cell body. Also, the mean $E$ of $7.6 \mathrm{kPa}$ in the cell body derived from MATLAB codes (Fig. 3E) was in consistence with that of $8.0 \mathrm{kPa}$ from the commercial offline software (Fig. 3B), embodying the reliability of the in-house developed MATLAB programs.

\section{DISCUSSION}

In this protocol, we elaborate how to run mechanical mapping of live cells using AFM in PF QNM mode. This method is practical as it empowers us to acquire global mechanical properties of a whole cell in high-speed with the same spatial resolution as that of surface topology scanning (Qi et al. 2017; Xu et al. 2019). Since the PF QNM mode can capture surface topography and mechanical properties synchronously, it can be used to correlate surface features with their mechanical properties in situ as shown in Fig. 3. The entire profile of surface mechanical properties in high spatial resolution also furthers our understanding on biological issues like cell transmigration, cell repolarization and cell mechanosensing under mechanical stimuli (Livne et al. 2014; Martinelli et al. 2014; Roduit et al. 2008; Schaefer and Hordijk 2015). This is especially meaningful by considering the heterogeneity of a cell with cell membrane, nucleus or nuclei, and numerous subcellular organelles. In this case, only one value of Young's modulus at a specific site of a cell is not able to represent the global mechanical features of that cell. 
The impacts of probe geometry on mechanical measurements should not be overlooked. In general, spherical probes are more stable than pyramid probes (Fig. 4). For pyramid probes, it is difficult to estimate precisely the parameters of tip-sample contact area, non-linear elastic responses and probe geometry calibration (like tip radius), which are important for $E$ fitting based on existing theories (Galluzzi et al. 2016). The paradox is that PF QNM mapping mode should use pyramid probes for high-resolution scanning. Thus, it needs to be emphasized that the acquired modulus data from PF QNM mode are contingent on probe geometries, fitting models, and scanning parameters. Several types of probes, like PFQNM-LC probes used in this protocol, are designed specifically for live cell mapping, and the main advantage is that this kind of probe suffers less hydrodynamic interference from high frequency excitation so as to favor more stable mechanical scanning (Schillers et al. 2016). Even using three distinct probes, the relative patterns of mechanical properties of HUVEC cultured on stiffness-varied substrates were similar, regardless of the differences in absolute $E$ values (Fig. 4).

In spite of the advantages in global and high-speed mechanical mapping of cell surface with high spatial resolution compared to the conventional ramp mode, there are several open issues to be clarified when applying PF QNM mode. The first issue is the high frequency excitation-induced cellular responses (HFEICR). Cellular viscoelasticity emerges obviously

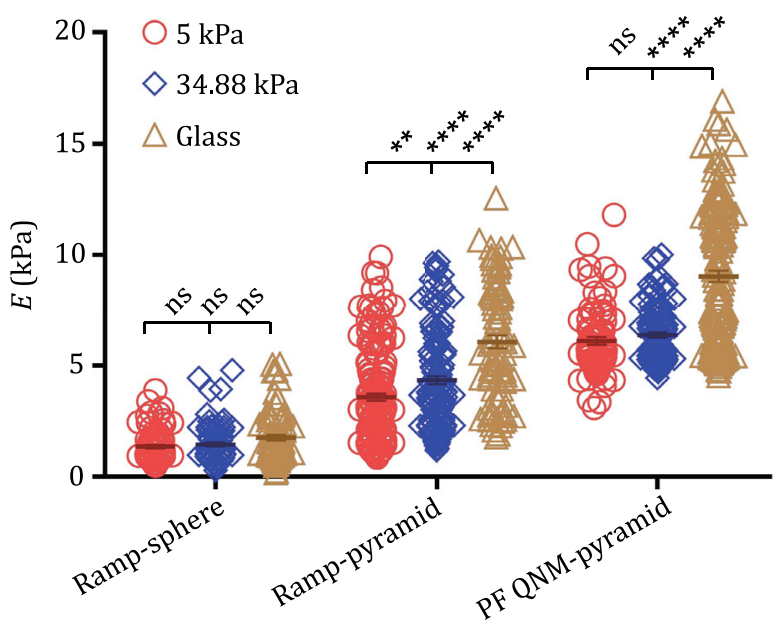

Fig. 4 Impacts of probe types and scanning modes on estimating mechanical properties. Young's modulus comparisons of HUVECs between ramp mode using spherical or pyramid probes and PF QNM mode using pyramid probe. The impacts of substrate stiffness on cellular mechanical property were also exhibited. Two-way ANOVA with Turkey's test was adopted, ns: no significant difference, ${ }^{* *} p<0.01$ and ${ }^{* * * *} p<0.0001$ when high frequency excitation is exerted on a cell, presenting the stress- or strain-induced cell stiffening or softening at a high frequency excitation (Chaudhuri et al. 2007; Gardel et al. 2004; Schmoller et al. 2010). It is noted that cellular stiffness upon PF QNM measurement is at most 100-fold higher than that upon the single-point ramp mode (Calzado-Martín et al. 2016). This huge difference can be minimized using the proper parameter settings for specific cell type. Actually, only one-fold increase of $E$ was found in HUVECs in this protocol than those using similar pyramid probes under ramp mode (Fig. 4), attributed to the application of the lower PF frequency setting of $0.25 \mathrm{kHz}$ and the usage of a PFQNM-LC probe.

Second issue arises from the bottom effect of cell culture substrate. No matter which type of probes/modes is adopted, most of culture substrates yields high stiffness, resulting in a rule of indentation depth/specimen thickness $<0.1$ when applying AFM indentation to soft material. To eliminate the potential artifacts stemming from the substrates, Bottom Effect Correction (BEC) was proposed theoretically (Gavara and Chadwick 2012), as a correction to Sneddon model when facing soft materials with pyramid or conical probes,

$$
\begin{aligned}
F= & \frac{8 E \tan \theta \delta^{2}}{3 \pi} \times\left\{1+1.7795 \frac{2 \tan \theta}{\pi^{2}} \frac{\delta}{h}\right. \\
& \left.+16(1.7795)^{2} \tan ^{2} \theta \frac{\delta^{2}}{h^{2}}+O\left(\frac{\delta^{3}}{h^{3}}\right)\right\},
\end{aligned}
$$

wherein $\theta$ is half-opening angle of the tip, $\delta$ is indentation depth and $h$ is specimen thickness. After obtaining cell height (Fig. 5A, pixel-to-pixel $h$ ) and indentation depth (Fig. 5B, pixel-to-pixel $\delta$ ) in PF QNM mapping, pixel-to-pixel BEC was done (Fig. 5C) by substituting pixel-to-pixel $\delta / h$ into Eq. 8 and the actual modulus distribution was obtained from the correction of pixel-to-pixel bottom effect. These typical results showed that BEC reduces those overestimated moduli at cell periphery. Here BEC was based on the accurate calculation of regional thickness (height) when glass bottom was used as zero plane for calibration of height. Evidently, the glass bottom calibration also limits the application of this method especially for fully spread, thin cells. In fact, only small fraction of the glass bottom or substrate can be imaged clearly when the cells grow up to high confluence, which could lead to invalidity of height calibration. In this case, AFM device combined with in situ confocal microscopy to monitor pixelto-pixel sample thickness might be a better choice to help determine the cell height with sliced confocal images. Alternatively, the bottom effect is less significant 

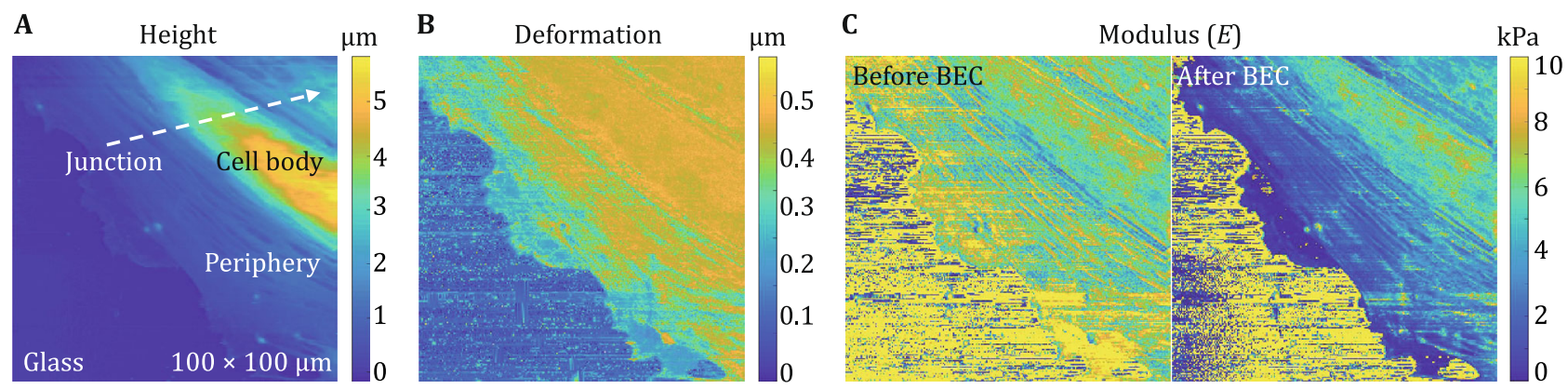

Fig. 5 Typical demonstration of bottom effect correction (BEC). A Height image. B Deformation image. C Modulus image before (left) and after (right) BEC. Glass substrate was set as zero plane for height calibration. Cell type: HUVEC

or even neglected when cells are seeded on soft substrate like PA gels with similar cellular stiffness and sufficient thickness, which will not cause large deviation (Gavara and Chadwick 2012).

Acknowledgements This work was supported by National Key Research and Development Program of China (2016YFA0501601), National Natural Science Foundation of China (31627804, 91642203, 31661143044, and 31570942), Frontier Science Key Project of Chinese Science Academy (QYZDJ-SSW-JSC018), and Strategic Priority Research Program of Chinese Academy of Sciences (XDB22040101).

\section{Compliance with Ethical Standards}

Conflict of interest Jinrong $\mathrm{Hu}$, Shenbao Chen, Dandan Huang, Yan Zhang, Shouqin Lü, and Mian Long declare that they have no conflict of interest.

Human and animal rights and informed consent This article does not contain any studies with human or animal subjects performed by any of the authors.

Open Access This article is licensed under a Creative Commons Attribution 4.0 International License, which permits use, sharing, adaptation, distribution and reproduction in any medium or format, as long as you give appropriate credit to the original author(s) and the source, provide a link to the Creative Commons licence, and indicate if changes were made. The images or other third party material in this article are included in the article's Creative Commons licence, unless indicated otherwise in a credit line to the material. If material is not included in the article's Creative Commons licence and your intended use is not permitted by statutory regulation or exceeds the permitted use, you will need to obtain permission directly from the copyright holder. To view a copy of this licence, visit http://creativecommons.org/ licenses/by/4.0/.

\section{References}

Åström KJ, Hägglund T (1995) PID controllers: theory, design, and tuning, vol 2. Instrument Society of America, Research Triangle Park
Barbee KA, Davies PF, Lal R (1994) Shear stress-induced reorganization of the surface topography of living endothelial cells imaged by atomic force microscopy. Circ Res 74:163-171

Calzado-Martín A, Encinar M, Tamayo J, Calleja M, San Paulo A (2016) Effect of actin organization on the stiffness of living breast cancer cells revealed by peak-force modulation atomic force microscopy. ACS Nano 10:3365-3374

Chaudhuri O, Parekh SH, Fletcher DA (2007) Reversible stress softening of actin networks. Nature 445:295-298

Curry N, Ghézali G, Kaminski Schierle GS, Rouach N, Kaminski CF (2017) Correlative STED and atomic force microscopy on live astrocytes reveals plasticity of cytoskeletal structure and membrane physical properties during polarized migration. Front Cell Neurosci 11:104

Derjaguin BV, Muller VM, Toporov YP (1975) Effect of contact deformations on the adhesion of particles. J Colloid Interf Sci 53:314-326

Efremov YM, Cartagena-Rivera AX, Athamneh AI, Suter DM, Raman A (2018) Mapping heterogeneity of cellular mechanics by multi-harmonic atomic force microscopy. Nat Protoc 13:2200

Galluzzi M, Biswas CS, Wu Y, Wang Q Du B, Stadler FJ (2016) Space-resolved quantitative mechanical measurements of soft and supersoft materials by atomic force microscopy. NPG Asia Mater 8:e327

Gardel M, Shin JH, MacKintosh F, Mahadevan L, Matsudaira P, Weitz D (2004) Elastic behavior of cross-linked and bundled actin networks. Science 304:1301-1305

Gavara N, Chadwick RS (2012) Determination of the elastic moduli of thin samples and adherent cells using conical atomic force microscope tips. Nat Nanotechnol 7:733-736

Geitmann A, Ortega JKE (2009) Mechanics and modeling of plant cell growth. Trends Plant Sci 14:467-478

Hansma HG, Kim KJ, Laney DE, Garcia RA, Argaman M, Allen MJ, Parsons SM (1997) Properties of biomolecules measured from atomic force microscope images: a review. J Struct Biol 119:99-108

Le S, Hu X, Yao M, Chen H, Yu M, Xu X, Nakazawa N, Margadant FM, Sheetz MP, Yan J (2017) Mechanotransmission and mechanosensing of human alpha-Actinin 1. Cell Rep 21:2714-2723

Liu H, Wen J, Xiao Y, Liu J, Hopyan S, Radisic M, Simmons CA, Sun Y (2014) In Situ mechanical characterization of the cell nucleus by atomic force microscopy. ACS Nano 8:3821-3828

Livne A, Bouchbinder E, Geiger B (2014) Cell reorientation under cyclic stretching. Nat Commun 5:3938

Long M, Sato M, Lim CT, Wu J, Adachi T, Inoue Y (2011) Advances in experiments and modeling in micro- and nanobiomechanics: a mini review. Cell Mol Bioeng 4:327-339 
Maivald P, Butt HJ, Gould SA, Prater CB, Drake B, Gurley JA, Elings VB, Hansma PK (1991) Using force modulation to image surface elasticities with the atomic force microscope. Nanotechnology 2:103-106

Marshall BT, Long M, Piper JW, Yago T, McEver RP, Zhu C (2003) Direct observation of catch bonds involving cell-adhesion molecules. Nature 423:190-193

Martinelli R, Zeiger AS, Whitfield M, Sciuto TE, Dvorak A, Van Vliet KJ, Greenwood J, Carman CV (2014) Probing the biomechanical contribution of the endothelium to lymphocyte migration: diapedesis by the path of least resistance. J Cell Sci 127:3720-3734

Ohashi T, Ishii Y, Ishikawa Y, Matsumoto T, Sato M (2002) Experimental and numerical analyses of local mechanical properties measured by atomic force microscopy for sheared endothelial cells. Bio-med Mat Eng 12:319-327

Pittenger B, Slade A (2013) Performing quantitative nanomechanical AFM measurements on live cells. Microsc Today 21:12-17

Qi J, Wu B, Feng S, Lü S, Guan C, Zhang X, Qiu D, Hu Y, Zhou Y, Li C, Long M, Jiao YL (2017) Mechanical regulation of organ asymmetry in leaves. Nat Plants 3:724

Radmacher M, Fritz M, Cleveland JP, Walters DA, Hansma PK (1994) Imaging adhesion forces and elasticity of lysozyme adsorbed on mica with the atomic force microscope. Langmuir 10:3809-3814

Rawlett AM, Hopson TJ, Nagahara LA, Tsui RK, Ramachandran GK, Lindsay SM (2002) Electrical measurements of a dithiolated electronic molecule via conducting atomic force microscopy. Appl Phys Lett 81:3043-3045

Rodriguez ML, McGarry PJ, Sniadecki NJ (2013) Review on cell mechanics: experimental and modeling approaches. Appl Mech Rev 65:060801-1-41
Roduit C, van der Goot FG, De Los Rios P, Yersin A, Steiner P, Dietler G, Catsicas S, Lafont F, Kasas S (2008) Elastic membrane heterogeneity of living cells revealed by stiff nanoscale membrane domains. Biophys J 94:1521-1532

Schaefer A, Hordijk PL (2015) Cell-stiffness-induced mechanosignaling - a key driver of leukocyte transendothelial migration. J Cell Sci 128:2221-2230

Schillers H, Medalsy I, Hu S, Slade AL, Shaw JE (2016) PeakForce Tapping resolves individual microvilli on living cells. J Mol Recognit 29:95-101

Schmoller K, Fernandez P, Arevalo R, Blair D, Bausch A (2010) Cyclic hardening in bundled actin networks. Nat Commun $1: 134$

Sneddon IN (1965) The relation between load and penetration in the axisymmetric Boussinesq problem for a punch of arbitrary profile. Int J Eng Sci 3:47-57

Sugimoto Y, Pou P, Abe M, Jelinek P, Pérez R, Morita S, Custance Ó (2007) Chemical identification of individual surface atoms by atomic force microscopy. Nature 446:64-67

Vargas-Pinto R, Gong H, Vahabikashi A, Johnson M (2013) The effect of the endothelial cell cortex on atomic force microscopy measurements. Biophys J 105:300-309

Wang J, Lü D, Mao D, Long M (2014) Mechanomics: an emerging field between biology and biomechanics. Protein Cell 5:518-531

Xu Y, Huang D, Lu S, Zhang Y, Long M (2019) Mechanical features of endothelium regulate cell adhesive molecule-induced calcium response in neutrophils. APL Bioeng 3:016104

Zhong Q Inniss D, Kjoller K, Elings VB (1993) Fractured polymer/ silica fiber surface studied by tapping mode atomic force microscopy. Surf Sci Lett 290:L688-L692 